\title{
Novel Coronavirus (COVID-19) Infection- Attributed Acute Pancreatitis: A Case Report and Literature Review
}

\author{
Rohit Chandra ${ }^{1}$, Nicholas J. Lazar ${ }^{1}$, Seth Goldman ${ }^{1}$, Zaid Imam ${ }^{2}$, Ramy Mansour ${ }^{3}$ \\ 1. Internal Medicine, Beaumont Health, Royal Oak, USA 2. Gastroenterology, Beaumont Medical Center, Royal Oak, \\ USA 3. Gastroenterology, Endoscopic Solutions PC, Clarkston, USA
}

Corresponding author: Rohit Chandra, rohitchandra2.0@gmail.com

\begin{abstract}
Novel coronavirus (COVID-19) has spread widely across the world inducing a global health crisis. Predominant signs of infection involve respiratory symptoms such as cough and dyspnea. Investigation into COVID-19 infection-associated gastrointestinal symptoms remains fluid. COVID-19-induced acute pancreatitis has been recorded from greater than 20 countries at this time. Herein, we submit a case of COVID-19-attributed acute pancreatitis, as well as a comprehensive assessment of previously reported cases of COVID-19-attributed acute pancreatitis.
\end{abstract}

Categories: Gastroenterology, Infectious Disease, Public Health

Keywords: acute pancreatitis, covid-19, viral pancreatitis, atlanta classification, sars-cov-2

\section{Introduction}

Gastrointestinal manifestations of the novel coronavirus (SARS-CoV-2) are common with 53\% of patients presenting with coronavirus infections (COVID-19) complaining of at least one gastrointestinal complaint, and $35 \%$ of patients presenting with mild elevations of aminotransferases and hyperbilirubinemia [1]. Data on pancreatic involvement in patients with COVID-19 is limited. A small cohort of 71 patients reported hyperlipasemia in nine patients and no cases of acute pancreatitis (AP). The hyperlipasemia in this cohort was attributed to subclinical pancreatic involvement or non-pancreatic hyperlipasemia [2]. We hereby report a case of COVID-19-attributed acute pancreatitis encountered on a review of hospitalized 1305 patients with COVID-19 and summarize the clinical presentation and outcomes of 26 other reported cases in the literature [3-25].

\section{Case Presentation}

A 53-year-old African American male presented in March 2020 with one week of progressively worsening shortness of breath and mild confusion. He also reported generalized abdominal pain for three days. His medical history included hypertension, benign prostatic hyperplasia, hyperlipidemia and bronchial asthma. His medications were triamterene, amlodipine, tamsulosin, atorvastatin and inhaled fluticasone. He had no

Review began 05/14/2021 Review ended 06/06/2021 Published 06/17/2021

\section{๑) Copyright 2021}

Chandra et al. This is an open access article distributed under the terms of the Creative Commons Attribution License CC-BY 4.0., which permits unrestricted use, distribution, and reproduction in any medium, provided the original author and source are credited. known history of diabetes mellitus or pancreatitis. He denied alcohol or tobacco use. He appeared with Kussmaul breathing, a respiratory rate of 40 breaths/minute, and an initial oxygen saturation of $99 \%$ on 3 liters of oxygen by nasal cannula.

Initial laboratory workup summarized in Table 1 demonstrated neutrophilic leukocytosis, severe hyperglycemia, high anion-gap metabolic acidosis, hyperlipasemia to $1200 \mathrm{U} / \mathrm{L}$ and acute kidney injury. Liver aminotransferases, and serum bilirubin were normal. A serum alcohol level was undetectable. These findings confirmed the diagnosis of severe diabetic ketoacidosis. A chest X-ray demonstrated bibasilar patchy infiltrates and a SARS-CoV-2 reverse transcriptase polymerase chain reaction (RT-PCR) was positive on a nasopharyngeal swab. The patient was intubated for respiratory failure in the setting of severe metabolic acidosis. High resolution computed tomography (CT) of the chest showed diffuse bibasilar atelectasis and patchy infiltrative changes (Figure 1). CT of the abdomen and pelvis demonstrated acute pancreatitis affecting the head and tail of the pancreas with no peripancreatic fluid collections or necrosis (Balthazar Grade C), with a normal gallbladder and biliary tract. Specifically, imaging showed peripancreatic fatty infiltrative changes that is seen about the head and tail of the pancreas (Figure 2). Aggressive fluid resuscitation, and intravenous insulin infusion were initiated. The patient received plaquenil, vitamin $\mathrm{C}$, and zinc for five days when ventilated allowing for extubation after receiving a total of 13 days of mechanical ventilation. Renal replacement therapy with continuous veno-venous hemodialysis was required for 20 days with subsequent renal recovery. Inflammatory markers were tested at admission and peaked on day 6 of admission and subsequently downtrended during admission. He subsequently recovered and was discharged after 30 days of hospitalization to a rehabilitation facility. On follow-up four months from hospitalization, he is doing well having recovered completely with no residual renal impairment, respiratory failure, and remains on insulin for diabetes. 


\section{Cureus}

\begin{tabular}{|c|c|c|c|}
\hline Laboratory Test & \multirow[t]{2}{*}{ Value } & \multirow{2}{*}{ Lab normal } & \multirow[t]{2}{*}{ Units } \\
\hline Complete Blood Count & & & \\
\hline Total White blood cell count & 20,300 & $3,500-10,100$ & Cells $/ \mathrm{mm}^{3}$ \\
\hline Neutrophil count & 16,100 & $1,600-7,200$ & Cells $/ \mathrm{mm}^{3}$ \\
\hline Lymphocyte Count & 1,400 & $1,100-4,000$ & Cells $/ \mathrm{mm}^{3}$ \\
\hline Platelet Count & 237,000 & $150,000-400,000$ & Cells $/ \mathrm{mm}^{3}$ \\
\hline Serum hemoglobin & 15.5 & 13.5-17.0 & $\mathrm{g} / \mathrm{dL}$ \\
\hline \multicolumn{4}{|l|}{ B. Serum chemistries } \\
\hline Albumin & 3.7 & $3.5-5.1$ & $\mathrm{~g} / \mathrm{dL}$ \\
\hline Sodium & 132 & 135-145 & $\mathrm{mEq} / \mathrm{L}$ \\
\hline Potassium & 5.9 & $3.5-5.2$ & $\mathrm{mEq} / \mathrm{L}$ \\
\hline Chloride & 99 & $98-111$ & $\mathrm{mEq} / \mathrm{L}$ \\
\hline Bicarbonate & 7 & $20-29$ & $\mathrm{mEq} / \mathrm{L}$ \\
\hline Serum glucose & 1291 & $60-99$ & $\mathrm{mg} / \mathrm{dL}$ \\
\hline Blood urea nitrogen & 89 & $7-25$ & $\mathrm{mg} / \mathrm{dL}$ \\
\hline Creatinine & 5.44 & $0.60-1.30$ & $\mathrm{mg} / \mathrm{dL}$ \\
\hline Lactic acid & 10.4 & $0.5-2.0$ & $\mathrm{mg} / \mathrm{dL}$ \\
\hline Beta hydroxybutyric acid & 11.92 & $0.02-0.27$ & $\mathrm{mmol} / \mathrm{L}$ \\
\hline Aspartate aminotransferase (AST) & 26 & $<35$ & $\mathrm{U} / \mathrm{L}$ \\
\hline Alanine aminotransferase (ALT) & 29 & $9-47$ & $U / L$ \\
\hline Total bilirubin & 0.3 & $0.3-1.2$ & $\mathrm{mg} / \mathrm{dL}$ \\
\hline Alcohol level & Undetectable & $<10$ & $\mathrm{mg} / \mathrm{dL}$ \\
\hline Lipase level & 1200 & $0-60$ & $U / L$ \\
\hline Triglyceride level & 677 & $0-149$ & $\mathrm{mg} / \mathrm{dL}$ \\
\hline \multicolumn{4}{|l|}{ C. Arterial blood gas } \\
\hline Arterial pH & 7.11 & - & - \\
\hline Serum pCO2 & 11 & - & $\mathrm{mmHg}$ \\
\hline Serum pO2 & 109 & - & $\mathrm{mmHg}$ \\
\hline Urine drug screen & Negative & - & - \\
\hline C-reactive protein (CRP) & 51.2 & & \\
\hline
\end{tabular}

TABLE 1: Summary of laboratory values on initial presentation 


\section{Cureus}

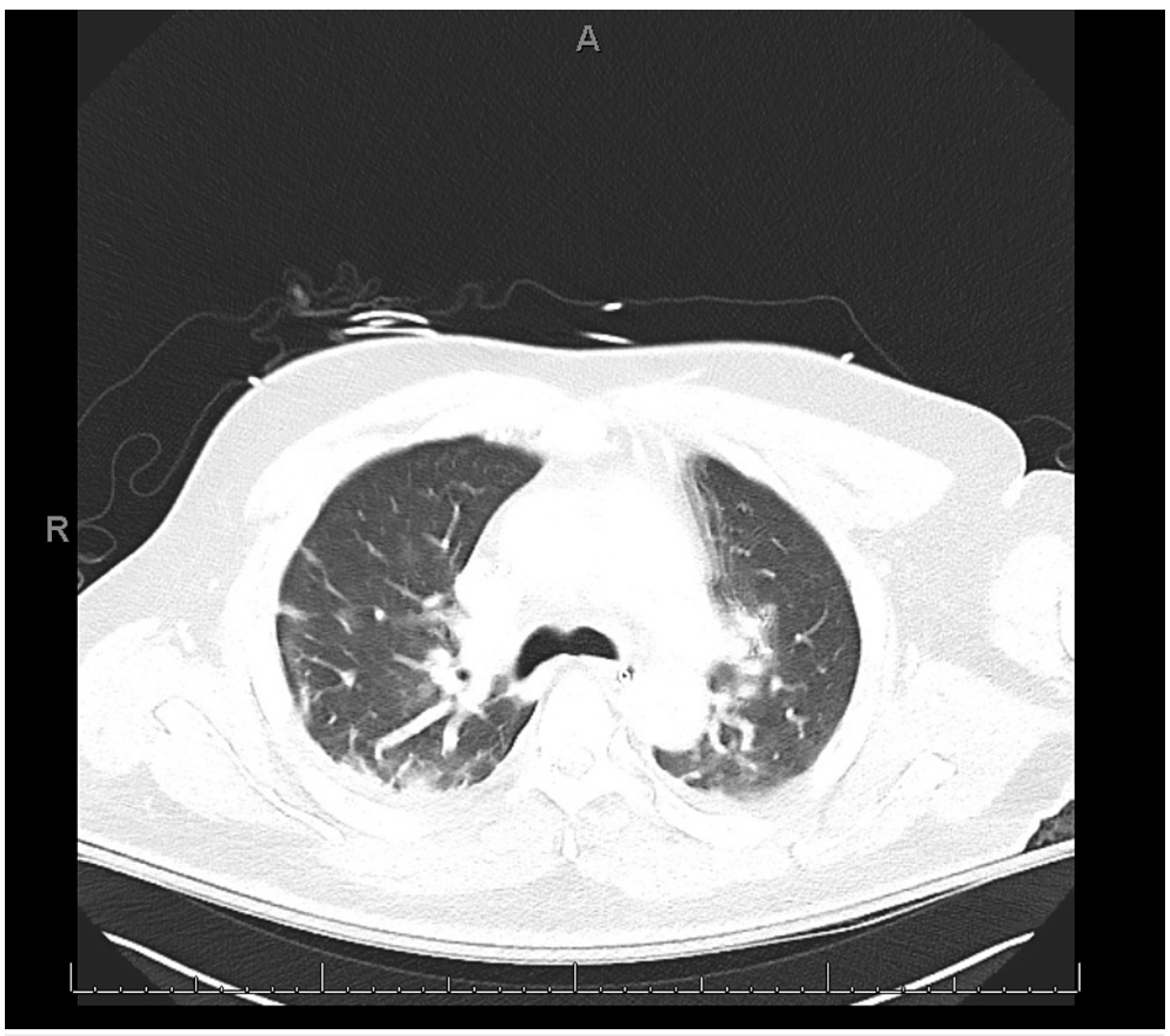

FIGURE 1: Computed tomography of chest

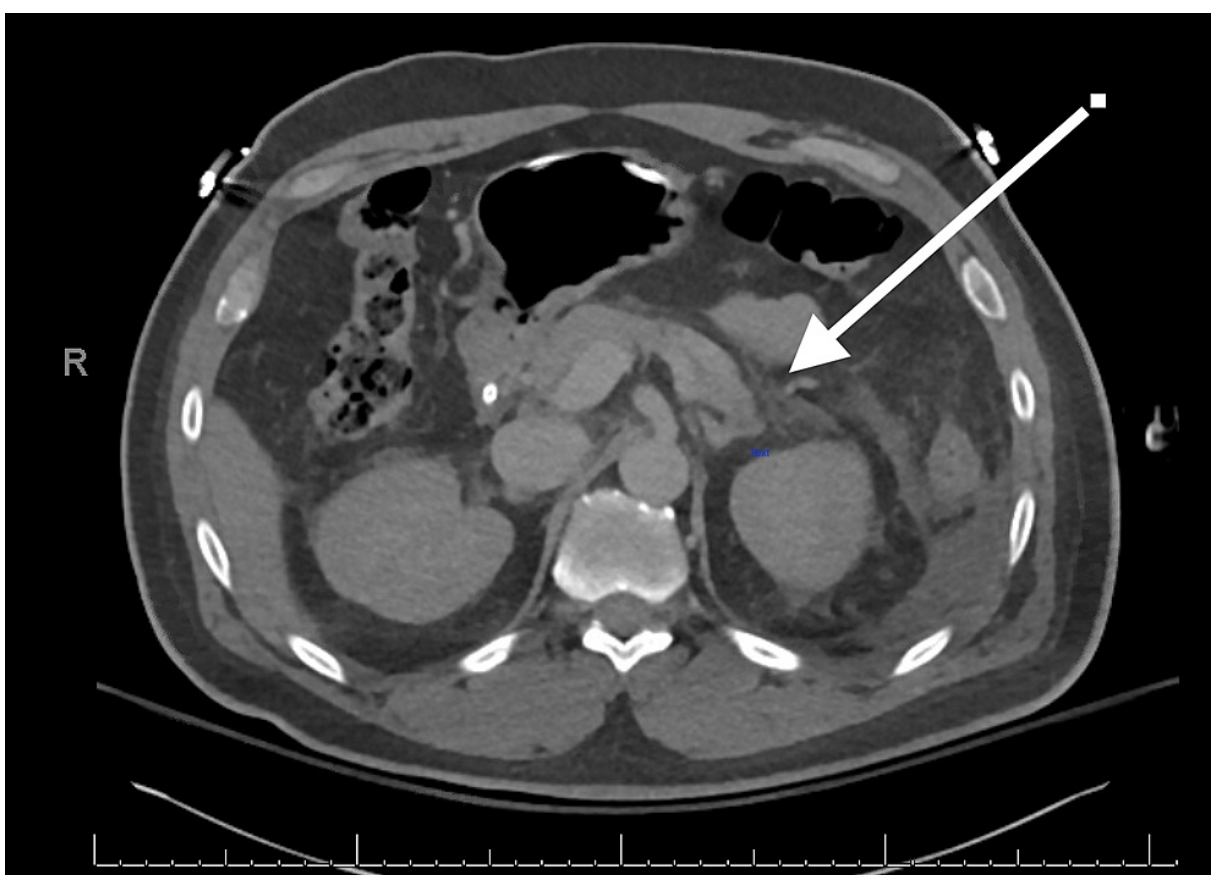

FIGURE 2: Computed tomography of the abdomen

\section{Discussion}

The novel coronavirus infection has been diagnosed in 95 million people and over 2 million deaths have been publicly recorded, and while its respiratory symptoms predominate clinical presentations, much has yet to be elucidated about its gastrointestinal complications including acute pancreatitis (AP). Initial cases of COVID-19-attributed AP were reported as early as June 2020 by Miao et al. [25]. Viral affinity to the 
pancreatic ACE-2 receptors and their activation have been proposed as a possible pathophysiological mechanism for COVID-19-attributed AP [2,26]. Other mechanisms including direct intra-acinar cellular replication similar to that resulting from hepatitis viruses are also possible [27].

Virus-attributed AP has been defined as the occurrence of AP as defined by the Revised Atlanta Classification (RAC), a temporal association with the implicated viral infection, and an exclusion of common etiologies of AP (biliary, alcohol, medication-induced, and metabolic causes) [27]. This definition is met by our reported case attributing AP to COVID-19. Organ failures (pulmonary and renal failure) occurring during the patient's admission appear more related to the severity of his diabetic ketoacidosis and acute respiratory distress syndrome (ARDS) resultant from the critical COVID-19 infection rather than the severity of his interstitial acute pancreatitis episode. Credence needs to be given to whether the reported patient could have developed AP secondary to diabetic ketoacidosis given prior reports on the association [28]. However, these associated cases typically exhibit severe hypertriglyceridemia greater than $1000 \mathrm{mg} / \mathrm{dL}$ or some degree of alcohol use, not seen in the reported patient [28-30].

To better discern the relationship between AP and COVID-19, we performed a review of PubMed and EMBASE databases for any published cases of COVID-19-attributed AP in adults since December 2019 as defined by their reporting authors. We then analyzed these cases to identify whether they met the definition of virus-attributed AP by Imam et al., in addition to the patients' clinical characteristics and outcomes [27].

A total of 26 cases were identified from 15 different countries with a median presenting age of 49.6 years [326]. Individual case characteristics are summarized in Table 2. Of published reports, all cases were temporally associated with COVID-19 infections and all cases met RAC definitions for AP diagnosis. Attempting to exclude other etiologies of acute pancreatitis, all but one case excluded alcohol use as an etiology, only 21 (80.8\%) patients had radiologic studies to exclude cholelithiasis, 15 (58\%) cases reported on evaluation for hypercalcemia, 19 (73\%) cases on excluding hypertriglyceridemia, and 16 (62\%) excluded drug-induced acute pancreatitis. This unfortunately introduces bias in published literature on the topic. An evaluation of the methodological quality of the case reports utilizing the Murad tool is summarized in Table 3. 


\section{Cureus}

\begin{tabular}{|c|c|c|c|c|c|c|c|c|c|c|}
\hline Author Name & Age (YRS)/Sex & Necrosis & Severity & Outcome & F/U & EtOH & Gallbladder Disease & Hypercalcemia & Medication Use & HyperTG \\
\hline Aloysius et al. [3] & $36 / \mathrm{F}$ & $\mathrm{N}$ & Severe & Recovery & 14 & $\mathrm{~N}$ & $\mathrm{~N}$ & - & $\mathrm{N}$ & $\mathrm{N}$ \\
\hline Kataria et al. [4] & 49/F & $\mathrm{N}$ & Severe & Recovery & 7 & $\mathrm{~N}$ & $\mathrm{~N}$ & $\mathrm{~N}$ & $\mathrm{~N}$ & $\mathrm{~N}$ \\
\hline Mazrouei et al. [15] & $24 / \mathrm{M}$ & $\mathrm{N}$ & Mild & Recovery & 3 & $\mathrm{~N}$ & $\mathrm{~N}$ & - & $\mathrm{N}$ & - \\
\hline Anand et al. [19] & $59 / \mathrm{F}$ & $\mathrm{N}$ & Mild & Recovery & 7 & $\mathrm{~N}$ & Cholecystectomy & - & $\mathrm{N}$ & $\mathrm{N}$ \\
\hline Kumaran et al. [20] & $67 / \mathrm{F}$ & $\mathrm{Y}$ & Severe & Recovery & 10 & $\mathrm{~N}$ & $\mathrm{~N}$ & $\mathrm{~N}$ & $\mathrm{~N}$ & $\mathrm{~N}$ \\
\hline Meireles et al. [21] & $36 / \mathrm{F}$ & $\mathrm{N}$ & Mild & Recovery & 3 & $\mathrm{~N}$ & $\mathrm{~N}$ & $\mathrm{~N}$ & $\mathrm{~N}$ & $\mathrm{~N}$ \\
\hline Cheung et al. [22] & 38/M & $\mathrm{N}$ & Mild & Recovery & 0 & $\mathrm{~N}$ & $\mathrm{~N}$ & $\mathrm{~N}$ & $\mathrm{~N}$ & $\mathrm{~N}$ \\
\hline Hadi et al. (1) [23] & $47 / \mathrm{F}$ & $\mathrm{N}$ & Severe & Recovery & 0 & $\mathrm{~N}$ & $\mathrm{~N}$ & - & - & $\mathrm{N}$ \\
\hline Hadi et al. (2) [23] & $68 / \mathrm{F}$ & $\mathrm{N}$ & Severe & Recovery & 22 & $\mathrm{~N}$ & - & $\mathrm{N}$ & - & $\mathrm{N}$ \\
\hline Hadi et al. (3) [23] & 71/M & $\mathrm{N}$ & Severe & Death & 0 & $\mathrm{~N}$ & - & - & - & - \\
\hline Rabice et al. [24] & $36 / \mathrm{F}$ & $\mathrm{N}$ & Moderate Severe & Recovery & 9 & $\mathrm{~N}$ & $\mathrm{~N}$ & $\mathrm{~N}$ & $\mathrm{~N}$ & $\mathrm{~N}$ \\
\hline Brikman et al. [5] & $61 / \mathrm{M}$ & $\mathrm{N}$ & Mild & Recovery & 2 & $\mathrm{~N}$ & $\mathrm{~N}$ & $\mathrm{~N}$ & $\mathrm{~N}$ & $\mathrm{~N}$ \\
\hline Bokhari and Mahmood [6] & $32 / \mathrm{M}$ & $\mathrm{N}$ & Mild & Recovery & 3 & $\mathrm{~N}$ & $\mathrm{~N}$ & $\mathrm{~N}$ & - & $\mathrm{N}$ \\
\hline Gonzalo-Voltas et al. [7] & $76 / \mathrm{F}$ & $\mathrm{N}$ & Mild & Recovery & 5 & $\mathrm{~N}$ & $\mathrm{~N}$ & - & - & $\mathrm{N}$ \\
\hline Gadiparthi et al. [8] & $40 / \mathrm{M}$ & $\mathrm{N}$ & Severe & Recovery & 6 & $\mathrm{~N}$ & $\mathrm{~N}$ & - & - & $\mathrm{Y}$ \\
\hline Karimzadeh et al. [9] & $65 / \mathrm{F}$ & $\mathrm{N}$ & Mild & Recovery & 18 & $\mathrm{~N}$ & - & - & - & - \\
\hline Wang et al. (1) [18] & $42 / \mathrm{M}$ & $\mathrm{N}$ & Severe & Mortality & 10 & $\mathrm{~N}$ & $\mathrm{~N}$ & $\mathrm{~N}$ & $\mathrm{~N}$ & $\mathrm{~N}$ \\
\hline Wang et al. (2) [18] & $35 / \mathrm{M}$ & $\mathrm{N}$ & Mild & Recovery & 18 & - & $\mathrm{N}$ & $\mathrm{N}$ & $\mathrm{N}$ & $\mathrm{N}$ \\
\hline Lakshmanan and Malik [10] & $68 / \mathrm{M}$ & $\mathrm{N}$ & Mild & Recovery & 7 & $\mathrm{~N}$ & $\mathrm{~N}$ & $\mathrm{~N}$ & $\mathrm{~N}$ & $\mathrm{~N}$ \\
\hline Kurihara et al. [11] & $55 / \mathrm{M}$ & $\mathrm{N}$ & Severe & Recovery & 26 & $\mathrm{~N}$ & $\mathrm{~N}$ & $\mathrm{~N}$ & $\mathrm{~N}$ & $\mathrm{~N}$ \\
\hline Dietrich et al. [12] & 72/M & $\mathrm{N}$ & Mild & Recovery & 59 & $\mathrm{~N}$ & $\mathrm{~N}$ & - & - & - \\
\hline Alves et al. [13] & $56 / \mathrm{F}$ & $\mathrm{N}$ & Severe & Recovery & 35 & $\mathrm{~N}$ & $\mathrm{~N}$ & $\mathrm{~N}$ & - & $\mathrm{N}$ \\
\hline Patnaik et al. [14] & $29 / M$ & $\mathrm{~N}$ & Mild & Recovery & 29 & $\mathrm{~N}$ & $\mathrm{~N}$ & - & - & $\mathrm{N}$ \\
\hline Purayil et al. [16] & $58 / \mathrm{M}$ & $\mathrm{N}$ & Mild & Recovery & 4 & $\mathrm{~N}$ & $\mathrm{~N}$ & - & $\mathrm{N}$ & - \\
\hline Miao et al. [25] & $26 / \mathrm{F}$ & $\mathrm{N}$ & Mild & Recovery & 7 & $\mathrm{~N}$ & - & $\mathrm{N}$ & $\mathrm{N}$ & $\mathrm{N}$ \\
\hline Kandasamy $[17]$ & $45 / \mathrm{F}$ & $\mathrm{N}$ & Mild & Recovery & 7 & $\mathrm{~N}$ & $\mathrm{~N}$ & $\mathrm{~N}$ & $\mathbf{N}$ & - \\
\hline
\end{tabular}

TABLE 2: Individual cases of COVID-19-attributed acute pancreatitis published in the literature HyperTG: Hypertriglyceridemia 


\section{Cureus}

\begin{tabular}{|c|c|c|c|c|c|c|c|c|c|c|c|c|c|}
\hline \multirow[t]{2}{*}{ Author Name } & $\mathbf{N}$ & Year & Q1 ${ }^{(1)}$ & & Q2 (2) & & Q3 ${ }^{\text {(3) }}$ & & Q4 ${ }^{(4)}$ & & $Q 5^{(5)}$ & & Risk of Bias \\
\hline & & & $\mathrm{Y}$ & $\mathrm{N}$ & $\mathrm{Y}$ & $\mathrm{N}$ & $\mathrm{Y}$ & $\mathrm{N}$ & $\mathrm{Y}$ & $\mathrm{N}$ & $\mathrm{Y}$ & $\mathrm{N}$ & \\
\hline Aloysius et al. [3] & 1 & 2020 & $\mathrm{Y}$ & & $\mathrm{Y}$ & & $\mathrm{Y}$ & & $\mathrm{Y}$ & & $\mathrm{Y}$ & & Low \\
\hline Kataria et al. [4] & 1 & 2020 & $\mathrm{Y}$ & & $\mathrm{Y}$ & & $\mathrm{Y}$ & & $\mathrm{Y}$ & & $\mathrm{Y}$ & & Low \\
\hline Mazrouei et al. [15] & 1 & 2020 & $\mathrm{Y}$ & & $\mathrm{Y}$ & & $\mathrm{Y}$ & & $\mathrm{Y}$ & & $\mathrm{Y}$ & & Low \\
\hline Anand et al. [19] & 1 & 2020 & $\mathrm{Y}$ & & $\mathrm{Y}$ & & $\mathrm{Y}$ & & $\mathrm{Y}$ & & $\mathrm{Y}$ & & Low \\
\hline Kumaran et al. [20] & 1 & 2020 & $\mathrm{Y}$ & & $\mathrm{Y}$ & & $\mathrm{Y}$ & & $\mathrm{Y}$ & & $\mathrm{Y}$ & & Low \\
\hline Meireles et al. [21] & 1 & 2020 & $\mathrm{Y}$ & & $\mathrm{Y}$ & & $\mathrm{Y}$ & & $\mathrm{Y}$ & & Y & & Low \\
\hline Cheung et al. [22] & 1 & 2020 & $\mathrm{Y}$ & & $\mathrm{Y}$ & & $\mathrm{Y}$ & & $\mathrm{Y}$ & & $\mathrm{Y}$ & & Low \\
\hline Hadi et al. [23] & 3 & 2020 & $\mathrm{Y}$ & & $\mathrm{Y}$ & & & $\mathrm{N}$ & $\mathrm{Y}$ & & $\mathrm{Y}$ & & Moderate \\
\hline Rabice et al. [24] & 1 & 2020 & $\mathrm{Y}$ & & $\mathrm{Y}$ & & $\mathrm{Y}$ & & $\mathrm{Y}$ & & $\mathrm{Y}$ & & Low \\
\hline Brikman et al. [5] & 1 & 2020 & $\mathrm{Y}$ & & $\mathrm{Y}$ & & $\mathrm{Y}$ & & $\mathrm{Y}$ & & $\mathrm{Y}$ & & Low \\
\hline Bokhari and Mahmood [6] & 1 & 2020 & $\mathrm{Y}$ & & $\mathrm{Y}$ & & $\mathrm{Y}$ & & $\mathrm{Y}$ & & $\mathrm{Y}$ & & Low \\
\hline Gonzalo-Voltas et al. [7] & 1 & 2020 & Y & & Y & & $\mathrm{Y}$ & & $\mathrm{Y}$ & & $\mathrm{Y}$ & & Low \\
\hline Gadiparthi et al. [8] & 1 & 2020 & $\mathrm{Y}$ & & $\mathrm{Y}$ & & & $\mathrm{N}$ & $\mathrm{Y}$ & & $\mathrm{Y}$ & & Moderate \\
\hline Karimzadeh et al. [9] & 1 & 2020 & $\mathrm{Y}$ & & $\mathrm{Y}$ & & & $\mathrm{N}$ & $\mathrm{Y}$ & & $\mathrm{Y}$ & & Moderate \\
\hline Wang et al. [18] & 2 & 2020 & $\mathrm{Y}$ & & $\mathrm{Y}$ & & $\mathrm{Y}$ & & $\mathrm{Y}$ & & $\mathrm{Y}$ & & Low \\
\hline Lakshmanan and Malik [10] & 1 & 2020 & $\mathrm{Y}$ & & $\mathrm{Y}$ & & $\mathrm{Y}$ & & $\mathrm{Y}$ & & $\mathrm{Y}$ & & Low \\
\hline Kurihara et al. [11] & 1 & 2020 & $\mathrm{Y}$ & & $\mathrm{Y}$ & & $\mathrm{Y}$ & & $\mathrm{Y}$ & & $\mathrm{Y}$ & & Low \\
\hline Dietrich et al. [12] & 1 & 2020 & $\mathrm{Y}$ & & $\mathrm{Y}$ & & & $\mathrm{N}$ & $\mathrm{Y}$ & & $\mathrm{Y}$ & & Moderate \\
\hline Alves et al. [13] & 1 & 2020 & $\mathrm{Y}$ & & $\mathrm{Y}$ & & $\mathrm{Y}$ & & $\mathrm{Y}$ & & Y & & Low \\
\hline Patnaik et al. [14] & 1 & 2020 & $\mathrm{Y}$ & & $\mathrm{Y}$ & & $\mathrm{Y}$ & & $\mathrm{Y}$ & & Y & & Low \\
\hline Purayil et al. [16] & 1 & 2020 & $\mathrm{Y}$ & & $\mathrm{Y}$ & & $\mathrm{Y}$ & & $\mathrm{Y}$ & & $\mathrm{Y}$ & & Low \\
\hline Miao et al. [25] & 1 & 2020 & $\mathrm{Y}$ & & $\mathrm{Y}$ & & $\mathrm{Y}$ & & $\mathrm{Y}$ & & $\mathrm{Y}$ & & Low \\
\hline Kandasamy [17] & 1 & 2020 & $Y$ & & $Y$ & & $Y$ & & $Y$ & & $\mathrm{Y}$ & & Low \\
\hline
\end{tabular}

\section{TABLE 3: Methodological quality assessment for included publications using the Murad tool}

(1) Did the patient(s) represent the whole case(s) of the medical center?

(2) Was the diagnosis correctly made?

(3) Were other important diagnoses excluded?

(4) Were all important data cited in the report?

(5) Was the outcome correctly ascertained?

* Meeting all five criteria renders the publication at low risk of bias, meeting four criteria at moderate risk of bias, and meeting three criteria at high risk of bias.

In terms of outcomes, mean length of follow-up was $11 \pm 13$ days and only one case of necrotizing pancreatitis was reported. Mortality occurred in two patients attributed to respiratory failure in both cases [18,23], while the remainder of patients survived their admission. Severity classification by RAC definitions was performed, identifying 10 (38.4\%) cases of severe AP, one (3.8\%) case of moderately severe $\mathrm{AP}$, and 15 (57.7\%) cases of mild AP. It is worthy to note given the high prevalence of organ failures in critical COVID-19 infections, these severity definitions are likely skewed by the severity of the primary 
infection (COVID-19) rather than the AP episode. Prior reports on hepatitis A and E-associated AP identified that outcomes were largely dependent on the occurrence of acute liver failure rather than the severity of the AP episode, suggesting the role of pancreatic involvement as a bystander organ [31,32]. This likely can be extrapolated in the setting of COVID-19-attributed AP as well.

\section{Conclusions}

With the ongoing COVID-19 pandemic, patients are presenting at emergency departments with a litany of different complaints. COVID-19-associated AP is infrequent and its role in affecting patient outcomes is unclear. Further investigation is needed to elucidate whether pancreatic involvement affects patient outcomes, and how best to manage these patients as aggressive resuscitation in patients with critical COVID-19 may worsen concurrent pulmonary pathology.

\section{Additional Information \\ Disclosures}

Human subjects: Consent was obtained or waived by all participants in this study. Conflicts of interest: In compliance with the ICMJE uniform disclosure form, all authors declare the following: Payment/services info: All authors have declared that no financial support was received from any organization for the submitted work. Financial relationships: All authors have declared that they have no financial relationships at present or within the previous three years with any organizations that might have an interest in the submitted work. Other relationships: All authors have declared that there are no other relationships or activities that could appear to have influenced the submitted work.

\section{References}

1. Elmunzer BJ, Spitzer RL, Foster LD, et al.: Digestive manifestations in patients hospitalized with coronavirus disease 2019 (PREPRINT). Clin Gastroenterol Hepatol. 2020, 10.1016/j.cgh.2020.09.041

2. McNabb-Baltar J, Jin DX, Grover AS, et al.: Lipase elevation in patients with COVID-19. Am J Gastroenterol. 2020, 115:1286-1288. 10.14309/ajg.0000000000000732

3. Aloysius MM, Thatti A, Gupta A, Sharma N, Bansal P, Goyal H: COVID-19 presenting as acute pancreatitis . Pancreatology. 2020, 20:1026-1027.10.1016/j.pan.2020.05.003

4. Kataria S, Sharif A, Ur Rehman A, Ahmed Z, Hanan A: COVID-19 induced acute pancreatitis: a case report and literature review. Cureus. 2020, 12:e9169. 10.7759/cureus.9169

5. Brikman S, Denysova V, Menzal H, Dori G: Acute pancreatitis in a 61-year-old man with COVID-19. CMAJ. 2020, 192:858-859. 10.1503/cmaj.201029

6. Bokhari SMMA, Mahmood F: Case report: Novel coronavirus-A potential cause of acute pancreatitis? . Am J Trop Med Hyg. 2020, 103:1154-1155. 10.4269/ajtmh.20-0568

7. Gonzalo-Voltas A, Fernández-Pérez-Torres CU, Baena-Díez JM: Acute pancreatitis in a patient with COVID19 infection (Article in English, Spanish). Med Clin (Barc). 2020, 155:183-184. 10.1016/j.medcli.2020.05.027

8. Gadiparthi C, Bassi M, Yegneswaran B, Ho S, Pitchumoni CS: Hyperglycemia, hypertriglyceridemia, and acute pancreatitis in COVID-19 infection: clinical implications. Pancreas. 2020, 49:62-63. 10.1097/MPA.0000000000001595

9. Karimzadeh S, Manzuri A, Ebrahimi M, Huy NT: COVID-19 presenting as acute pancreatitis: lessons from a patient in Iran. Pancreatology. 2020, 20:1024-1025. 10.1016/j.pan.2020.06.003

10. Lakshmanan S, Malik A: Acute pancreatitis in mild COVID-19 infection . Cureus. 2020, 12:e9886. 10.7759/cureus.9886

11. Kurihara Y, Maruhashi T, Wada T, Osada M, Oi M, Yamaoka K, Asari Y: Pancreatitis in a patient with severe coronavirus disease pneumonia treated with veno-venous extracorporeal membrane oxygenation. Intern Med. 2020, 59:2903-2906. 10.2169/internalmedicine.5912-20

12. Dietrich CG, Hübner D, Marx G, Bickenbach J, Bootsveld A: Primary presentation of COVID-19 solely with gastrointestinal symptoms: a problem for the containment of the disease. Eur J Gastroenterol Hepatol. 2020, 32:1475-1478. 10.1097/MEG.0000000000001922

13. Alves AM, Yvamoto EY, Marzinotto MAN, Teixeira ACS, Carrilho FJ: SARS-CoV-2 leading to acute pancreatitis: an unusual presentation. Braz J Infect Dis. 2020, 24:561-564. 10.1016/j.bjid.2020.08.011

14. Patnaik RNK, Gogia A, Kakar A: Acute pancreatic injury induced by COVID-19. IDCases. 2020, 22:e00959. 10.1016/j.idcr.2020.e00959

15. Mazrouei SSA, Saeed GA, Al Helali AA: COVID-19-associated acute pancreatitis: a rare cause of acute abdomen. Radiol Case Rep. 2020, 15:1601-1603. 10.1016/j.radcr.2020.06.019

16. Purayil N, Sirajudeen J, Va N, Mathew J: COVID-19 presenting as acute abdominal pain: a case report . Cureus. 2020, 12:e9659. 10.7759/cureus.9659

17. Kandasamy S: An unusual presentation of COVID-19: acute pancreatitis . Ann Hepatobiliary Pancreat Surg. 2020, 24:539-541. 10.14701/ahbps.2020.24.4.539

18. Wang K, Luo J, Tan F, et al.: Acute pancreatitis as the initial manifestation in 2 cases of COVID-19 in Wuhan, China. Open Forum Infect Dis. 2020, 7:ofaa324. 10.1093/ofid/ofaa324

19. Anand ER, Major C, Pickering O, Nelson M: Acute pancreatitis in a COVID-19 patient . Br J Surg. 2020, 107:e182. 10.1002/bjs.11657

20. Kumaran NK, Karmakar BK, Taylor OM: Coronavirus disease-19 (COVID-19) associated with acute necrotising pancreatitis (ANP). BMJ Case Rep. 2020, 13:e237903. 10.1136/bcr-2020-237903

21. Meireles PA, Bessa F, Gaspar P, Parreira I, Silva VD, Mota C, Alvoeiro L: Acalculous acute pancreatitis in a COVID-19 patient. Eur J Case Rep Intern Med. 2020, 7:001710. 10.12890/2020 001710

22. Cheung S, Fuentes AD, Fetterman AD: Recurrent acute pancreatitis in a patient with COVID-19 infection . 


\section{Cureus}

Am J Case Rep. 2020, 21 :e927076. 10.12659/AJCR.927076

23. Hadi A, Werge M, Kristiansen KT, Pedersen UG, Karstensen JG, Novovic S, Gluud LL: Coronavirus disease-19 (COVID-19) associated with severe acute pancreatitis: case report on three family members. Pancreatology. 2020, 20:665-667. 10.1016/j.pan.2020.04.021

24. Rabice SR, Altshuler PC, Bovet C, Sullivan C, Gagnon AJ: COVID-19 infection presenting as pancreatitis in a pregnant woman: a case report. Case Rep Women's Health. 2020, 27:e00228. 10.1016/j.crwh.2020.e00228

25. Miao Y, Lidove O, Mauhin W: First case of acute pancreatitis related to SARS-CoV-2 infection . Br J Surg. 2020, 107:e270. 10.1002/bjs.11741

26. Wang F, Wang H, Fan J, Zhang Y, Wang H, Zhao Q: Pancreatic injury patterns in patients with coronavirus disease 19 pneumonia. Gastroenterology. 2020, 159:367-370. 10.1053/j.gastro.2020.03.055

27. Imam Z, Simons-Linares CR, Chahal P: Infectious causes of acute pancreatitis: a systematic review . Pancreatology. 2020, 20:1312-1322. 10.1016/j.pan.2020.08.018

28. Nair S, Yadav D, Pitchumoni CS: Association of diabetic ketoacidosis and acute pancreatitis: observations in 100 consecutive episodes of DKA. Am J Gastroenterol. 2000, 95:2795-2800. 10.1111/j.15720241.2000.03188.x

29. Seth A, Rajpal S, Saigal T, et al.: Diabetic ketoacidosis-induced hypertriglyceridemic acute pancreatitis treated with plasmapheresis-recipe for biochemical disaster management. Clin Med Insights Gastroenterol. 2014, 7:51-53. 10.4137/CGast.S18557

30. Kumar P, Sakwariya A, Sultania AR, Dabas R: Hypertriglyceridemia-induced acute pancreatitis with diabetic ketoacidosis: a rare presentation of type 1 diabetes mellitus. J Lab Physicians. 2017, 9:329-331. 10.4103/JLP.JLP 53 17

31. Haffar S, Bazerbachi F, Garg S, Lake JR, Freeman ML: Frequency and prognosis of acute pancreatitis associated with acute hepatitis E: a systematic review. Pancreatology. 2015, 15:321-326. 10.1016/j.pan.2015.05.460

32. Haffar S, Bazerbachi F, Prokop L, Watt KD, Murad MH, Chari ST: Frequency and prognosis of acute pancreatitis associated with fulminant or non-fulminant acute hepatitis A: a systematic review. Pancreatology. 2017, 17:166-175. 10.1016/j.pan.2017.02.008 